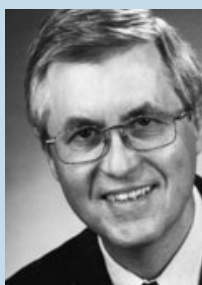

Prof. Dr. rer. nat. Jürgen Kießling Universitätsklinikum Gießen

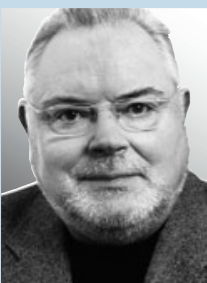

Dr. med.

Dieter Leithäuser

HNO-Arzt, Warburg

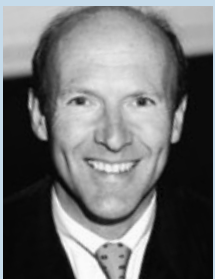

Prof. Dr. med. Ralph Mösges Universität Köln dagegen 14,7\% Komplikationen mit 1,9\% schweren Komplikationen. Kinder hatten Wundinfektionen, Gleichgewichtsstörungen und Blutergüsse, während die Erwachsenen häufiger neben Gleichgewichtsstörungen über Geschmacksstörungen berichteten.

Die Vergleichbarkeit in der Qualität der Versorgung mit Cochlea-Implantaten, die mit den Komplikationsraten beginnt und eigentlich mit einer Erfolgskontrolle enden sollte, ist sicherlich für viele Interessengruppen auch wegen der möglichen auszuübenden
Kontrolle ein spannendes Thema. International üblich ist wegen dieser gemischten Interessenlage daher, eine "antizipierte", d.h. konsensfähige und qualitätssichernde Übereinkunft à la Leitlinie zu schaffen, in das die Erfahrungen und Erkenntnisse verschiedenster Implantat- und Interessen-Gruppen einfließen können. So einfach wird sich daher die Publikation nicht durchsetzen, da ihr zusätzlich der „bias“ eines einzelnen Zentrums anhaftet und andere Zentren je nach Krankengut und Selektion andere
Verteilungen haben werden. Eine deutsche Leitlinie "Cochlea-Implant" ist übrigens bei der AWMF angemeldet. Als Zielorientierung ist u.a. angekündigt: Qualitätssicherung der Cochlea-Implant-Versorgung.

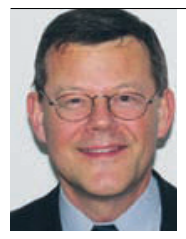

Prof. Dr. med. Olaf Michel Universitair Ziekenhuis, Brüssel

\section{Hyperfraktionierte Radiatio vielversprechend}

Bieten Bestrahlungsregimes mit kürzerer Behandlungszeit Vorteile gegenüber der konventionellen Radiatio? Autoren einer großen multizentrisch durchgeführten Phase-III-Studie prüften dies bei Karzinomen im Kopf-Hals-Bereich. $\mathrm{n}$ der Studie verglich das britische Team bei 918 Patienten mit fortgeschrittenen Plattenepithelkarzinomen im Kopf-Hals-Bereich ein verkürztes Bestrahlungsregime mit der konventionellen Radiatio. T1M0-Tumoren der Mundhöhle, des Rachenraums und des

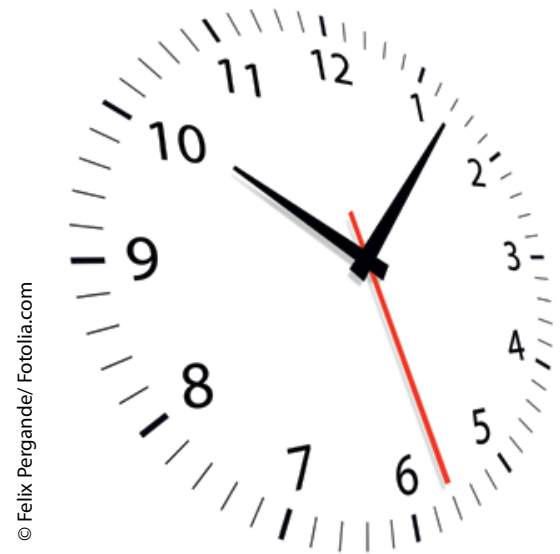

Kehlkopfs waren ausgeschlossen, Patienten mit Fernmetastasen ebenfalls.

Die Patienten erhielten in der ersten Gruppe eine kontinuierliche hyperfraktionierte Bestrahlung mit verkürztem Zeitintervall (continuous hyperfractionated accelerated radiotherapy, CHART): 1,5 Gy pro Fraktion, drei Fraktionen pro Tag kontinuierlich über zwölf Tage mit mindestens sechs Stunden Pause zwischen den einzelnen Fraktionen; die Gesamtdosis betrug 54 Gy. In der Kontrollgruppe wurde eine herkömmliche Bestrahlung mit fünf täglichen Bestrahlungsfraktionen zu 2 Gy pro Woche über sechseinhalb Wochen durchgeführt. Die Dosis lag hier bei 66 Gy.

Bei bis zu zwölf Jahren Beobachtungszeit lag die 10-Jahresrate für lokoregionäres rezidivfreies Überleben in der CHART-Gruppe bei $43 \%$, in der Kontrollgruppe bei $50 \%$. Dieser Unterschied war mit $p=0,2$ ebenso wenig signifikant wie bei Gesamtüberleben ( 26 vs. $29 \%$, p = 0,4 ), krankheitsfreiem (41 vs. $46 \%, \mathrm{p}=$ $0,3)$ und krankheitsspezifischem Überleben ( 56 vs. $58 \%, \mathrm{p}=0,5$ ).

Bei den unerwünschten Nebenwirkungen zeigten sich für leichte wie schwere Ereignisse signifikante Vorteile von CHART. Dies galt insbesondere für noch zehn Jahre nach der Bestrahlung existierende schwere Xerostomien (23 vs. $31 \%$, $\mathrm{p}=0,02$ ), Schleimhautnekrosen (5 vs. $9 \%$, $\mathrm{p}=0,02$ ) und Larynxödeme (50 vs. $60 \%$, $\mathrm{p}=0,05)$.

Fazit: In dieser großen Phase-III-Studie ergaben sich trotz reduzierter Strahlendosis keine Überlebensnachteile für das hyperfraktionierte Bestrahlungsregime. Diverse schwere Langzeitnebenwirkungen waren unter dieser Therapie deutlich vermindert.
Saunders Ml et al. Mature results of a randomized trial of accelerated hyperfractionated versus conventional radiotherapy in headand-neck cancer. Int J Radiat Oncol Biol Phys 2010; 77: 3-8. 